\title{
Likelihood Ratio Gradient Estimation for Meixner Distribution and Lévy Processes
}

\author{
REIICHIRO KAWAI
}

\begin{abstract}
We address the problem of gradient estimation with respect to four characterizing parameters of the Meixner distribution and Lévy process. With the help of the explicit marginal probability density function, the likelihood ratio method is directly applicable, while unbiased estimators may contain infinite random series in their score function. We quantify the estimator bias arising when the infinite series is truncated to finite term. We further propose a substantially simple exact simulation method for the Meixner distribution, based on acceptance-rejection sampling and the Esscher density transform. Numerical results are presented in the context of financial Greeks to illustrate the effectiveness of our formulas along with bias estimates.

Keywords: Acceptance-rejection sampling; Esscher density transform; Greeks; Lévy process; likelihood ratio method; Meixner distribution; Meixner process; Monte Carlo simulation.

2010 Mathematics Subject Classification: 62F12, 91B70, 60E07, 60G51.
\end{abstract}

\section{Introduction}

Estimation of parameter sensitivity of expectations has been a central issue in analysis of stochastic models. Three methods have widely been known for this purpose; the finite difference method, the pathwise method and the likelihood ratio method. (See, for example, Glasserman [4] for their details.) They are either applicable or inapplicable depending on different model characteristics in different settings under consideration. Among those, the likelihood ratio method along integration-by-parts in the sense of Malliavin calculus is known to be useful for deriving unbiased estimators. The first result in this direction is [3] in deriving financial Greeks formulas, which correspond to gradient of an expected value with respect to its characterizing parameters. The integration-by-parts technique cannot simply be applied to the setting with the presence of Poisson jumps in a similar manner. All the existing results on the computation of Greeks on Poisson space, except for [2], has to employ some techniques to avoid the naive use of Malliavin calculus on Poisson space. The Malliavin calculus on Wiener space is applied in [11] to the time-changed Brownian motion, such as variance gamma and inverse Gaussian Lévy processes, conditionally on the time-changing process. In [12, 13, 14], the scaling property of gamma processes with respect to the Girsanov transform is used to derive Greeks for asset price dynamics described by gamma, stable and tempered stable processes. Some approximative, yet broadly applicable, approaches have also been discussed in similar Lévy-driven settings in [5, 6] to obtain estimators through the explicit characteristic function and simulation methods.

In this paper, we address the problem of gradient estimation with respect to four characterizing parameters of the Meixner distribution and Lévy process. The Meixner process has been recognized as a successful class of Lévy processes for the purpose of practical modeling, such as mathematical finance and possibly turbulence, as well as is of theoretical interest. One of the remarkable properties of the Meixner process is its asymptotic behaviors with respect to observation time; in a short time frame it approximates an Cauchy Lévy process, while over long intervals it is close to a Brownian motion. These time behaviors of stable-type and Gaussian-type have long been considered to be very appealing in various applications. (See, for example, [7, 17, 19], to mention just a few. Also, some simulation and statistical aspects of the Meixner process are discussed in [8, 10, 15].) In the context of sensitivity analysis, to the best of our knowledge, the approach of integration-by-parts type is either not applicable in general (applicable only for a special set of parameters [20] with the help of the scaling property gamma random variables [12]) or not very practical (the approach of [11] can be employed for Meixner processes as a time-changed Brownian motion [17], while estimators are very difficult to simulate.) In this paper, with the help of the explicit marginal probability density function, we apply the likelihood ratio method to obtain unbiased estimators for sensitivity indices in a straightforward manner. One of the remarkable properties of the

Published in Computational Statistics. doi:10.1007/s00180-011-0288-7. Email Address: reiichiro.kawai@le.ac.uk. Postal Address: Department of Mathematics, University of Leicester, Leicester LE1 7RH, UK. The author would like to thank the anonymous referee for various helpful comments. 
Meixner process is the availability of a relatively simple marginal probability density function in closed form, unlike the normal inverse Gaussian process in which modified Bessel functions are involved. Unlike the aforementioned results obtained through Malliavin calculus, however, (most of) our unbiased estimators are not truly unbiased, from a practical point of view, in the sense that their likelihood ratio may contain infinite random series. With a view towards practical use, we analyze the estimator bias arising when the infinite random series is truncated to finite term. Moreover, we propose a substantially simple exact simulation method for arbitrary Meixner distributions for Monte Carlo estimation purposes. Our simulation method is based on acceptance-rejection sampling of [1] and the Esscher density transform, with which sensitivity index can be estimated solely through generating uniform random variables and evaluating the Meixner probability density function, without sampling directly from the density function.

The rest of this paper is organized as follows. Section 2 is devoted to a review of basic facts on the Meixner distribution and Lévy processes, which we use throughout this paper. In Section 3.1, we derive unbiased estimators for parameter sensitivity indices on the Meixner distribution and Lévy process. The derivation of our formulas entails rather lengthy proofs of somewhat routine nature. To avoid overloading the paper, we omit nonessential details in some instances. In Section 3.2, a simple exact simulation method for the Meixner distribution is proposed for Monte Carlo estimation purposes. Some numerical results are presented in Section 4 in the context of Monte Carlo estimation of financial Greeks to illustrate the effectiveness of our formulas along with bias bounds.

\section{Preliminaries}

Let us begin this preliminary section with the notations which will be used throughout the paper. We denote by $\mathbb{R}^{d}$ the $d$-dimensional Euclidean space with the norm $\|\cdot\|$, and $|\cdot|$ for one-dimension. We use the notation $i:=\sqrt{-1}$ and let $\mathbb{N}$ denote the collection of positive integers. We denote by $\top$ the transpose. We fix $(\Omega, \mathscr{F}, \mathbb{P})$ as our underlying probability space. We denote the Gamma function by $\Gamma(z):=\int_{0}^{+\infty} x^{z-1} e^{-x} d x$, where $z$ is a complex number with a positive real part.

The Meixner distribution, denoted by $\operatorname{Meixner}(\alpha, \beta, \delta, \mu)$, is infinitely divisible and selfdecomposable with $\alpha>0$, $|\beta|<\pi, \delta>0, \mu \in \mathbb{R}$. Throughout this paper, we write $\theta:=(\alpha, \beta, \delta, \mu)$. The Meixner $(\alpha, \beta, \delta, \mu)$ distribution has the explicit characteristic function

$$
\varphi(y ; \theta):=e^{i y \mu}\left(\frac{\cos (\beta / 2)}{\cosh ((\alpha y-i \beta) / 2)}\right)^{2 \delta}, \quad y \in \mathbb{R}
$$

and admits the probability density $f(x ; \theta):=f(x ; \alpha, \beta, \delta, \mu)$ given in closed form

$$
f(x ; \theta):=\frac{(2 \cos (\beta / 2))^{2 \delta}}{2 \pi \alpha \Gamma(2 \delta)} \exp \left[\frac{\beta}{\alpha}(x-\mu)\right]\left|\Gamma\left(\delta+i \frac{x-\mu}{\alpha}\right)\right|^{2}, \quad x \in \mathbb{R},
$$

with the so-called semi-heavy tails

$$
f(x ; \theta) \sim|x|^{2 \delta-1} \exp \left[-\frac{\pi \pm \beta}{\alpha}|x|\right], \quad \text { as } x \rightarrow \pm \infty .
$$

The Lévy measure of Meixner $(\alpha, \beta, \delta, \mu)$ admits the Lebesgue density

$$
g(z)=\delta \frac{\exp (\beta z / \alpha)}{z \sinh (\pi z / \alpha)}, \quad z \in \mathbb{R} \backslash\{0\} .
$$

Loosely speaking, $\alpha$ stands for the tail heaviness, $\beta$ the skew, $\delta$ the scale, and $\mu$ the location. Let $\left\{X_{t}: t \geq 0\right\}$ be a Lévy process such that $\mathscr{L}\left(X_{1}\right)=\operatorname{Meixner}(\alpha, \beta, \delta, \mu)$. This Lévy process is called the Meixner process. With the aid of (2.1), it holds that for each $\sigma>0$ and $t>0$,

$$
\mathscr{L}\left(\sigma X_{t}\right)=\operatorname{Meixner}(\sigma \alpha, \beta, t \delta, t \sigma \mu) .
$$

One of the remarkable properties of the Meixner process is its asymptotic behaviors with respect to observation time, just like normal inverse Gaussian processes, tempered stable processes of Rosiński [18] and layered stable processes of Houdré and Kawai [9]. On the one hand, over short time intervals, it approximates a stable process; as $h \downarrow 0$, a scaled Meixner process

$$
\left\{\frac{1}{h \alpha \delta}\left(X_{h t}-h t \mu\right): t \geq 0\right\}
$$


tends to a standard Cauchy (Lévy) process, where the convergence is in the weak sense of random processes in the space of càdlàg functions from $[0,+\infty)$ into $\mathbb{R}$ equipped with the Skorohod topology. In a long time frame, on the other hand, it is close to a Brownian motion; as $h \uparrow+\infty$, another scaled Meixner process

$$
\left\{\frac{\cos (\beta / 2)}{\alpha} \sqrt{\frac{2}{h \delta}}\left(X_{h t}-h t(\mu+\alpha \delta \tan (\beta / 2))\right): t \geq 0\right\}
$$

approaches to the standard Brownian motion. These stable-type and Gaussian-type behaviors have long been considered to be very appealing in various applications, for example, to model turbulence in statistical physics, asset price dynamics in mathematical finance, network dynamics in transportation management and computer science, and animal movement patterns in mathematical biology. (Those can be proved rigorously in a similar manner to [9, 18].)

\section{Main Results}

Throughout this section, we let $X$ be a random variable such that $\mathscr{L}(X) \sim \operatorname{Meixner}(\alpha, \beta, \delta, \mu)$ and let $\Phi$ be a measurable function mapping from $\mathbb{R} \rightarrow \mathbb{R}$ such that

$$
\mathbb{E}_{\theta}[|\Phi(X)|(1 \vee|X|)]<+\infty
$$

where $\mathbb{E}_{\theta}$ indicates the expectation taken under the $\operatorname{Meixner}(\alpha, \beta, \delta, \mu)$ distribution. For convenience, we use the notation

$$
\widetilde{X}:=\frac{X-\mu}{\alpha}
$$

It is important that no regularity of the function $\Phi$ is not imposed, as we apply the likelihood ratio method.

\subsection{Estimation Formulas and Error Analysis}

For each $n \in \mathbb{N}$, define

$$
\begin{aligned}
& \Xi_{n}^{(1)}(\Phi, \theta):=\frac{1}{\alpha} \mathbb{E}_{\theta}\left[\Phi(X)\left(-1-\beta \widetilde{X}+\sum_{k=0}^{n-1} \frac{2 \widetilde{X}^{2}}{(k+\delta)^{2}+\widetilde{X}^{2}}+2|\widetilde{X}|\left(\frac{\pi}{2}-\tan ^{-1} \frac{n+\delta}{|\widetilde{X}|}\right)\right)\right] \\
& \Xi^{(2)}(\Phi, \theta):=\mathbb{E}_{\theta}\left[\Phi(X)\left(\widetilde{X}-\delta \tan \frac{\beta}{2}\right)\right] \\
& \Xi_{n}^{(3)}(\Phi, \theta):=\mathbb{E}_{\theta}\left[\Phi(X)\left(2 \ln (2 \cos (\beta / 2))+2 \sum_{k=0}^{n-1}\left(\frac{1}{k+2 \delta}-\frac{k+\delta}{(k+\delta)^{2}+\widetilde{X}^{2}}\right)-\ln \frac{(n+\delta)^{2}+\widetilde{X}^{2}}{(n+2 \delta)^{2}}\right)\right] \\
& \Xi_{n}^{(4)}(\Phi, \theta):=\frac{1}{\alpha} \mathbb{E}_{\theta}\left[\Phi(X)\left(-\beta+\sum_{k=0}^{n-1} \frac{2 \widetilde{X}}{(k+\delta)^{2}+\widetilde{X}^{2}}+2 \operatorname{sgn}(\widetilde{X})\left(\frac{\pi}{2}-\tan ^{-1} \frac{n+\delta}{|\widetilde{X}|}\right)\right)\right]
\end{aligned}
$$

Note that the condition (3.1) ensures that for each $n \in \mathbb{N}$, the expectations (3.2)-(3.5) are all well defined. The following provides unbiased estimators for sensitivity index with bias estimates.

Theorem 3.1. It holds that for each $n \in \mathbb{N}$,

$$
\begin{aligned}
\left|\frac{\partial}{\partial \alpha} \mathbb{E}_{\theta}[\Phi(X)]-\Xi_{n}^{(1)}(\Phi, \theta)\right| & <\frac{2}{\alpha} \mathbb{E}_{\theta}\left[|\Phi(X)| \frac{\widetilde{X}^{2}}{(n+\delta)^{2}+\widetilde{X}^{2}}\right], \\
\left|\frac{\partial}{\partial \delta} \mathbb{E}_{\theta}[\Phi(X)]-\Xi_{n}^{(3)}(\Phi, \theta)\right| & <\frac{2}{n+\delta} \mathbb{E}_{\theta}\left[|\Phi(X)|\left(\frac{\delta}{n+2 \delta} \wedge \frac{\widetilde{X}^{2}}{(n+\delta)^{2}+\widetilde{X}^{2}}\right)\right], \\
\left|\frac{\partial}{\partial \mu} \mathbb{E}_{\theta}[\Phi(X)]-\Xi_{n}^{(4)}(\Phi, \theta)\right| & <\frac{2}{\alpha} \mathbb{E}_{\theta}\left[|\Phi(X)| \frac{|\widetilde{X}|}{(n+\delta)^{2}+\widetilde{X}^{2}}\right] .
\end{aligned}
$$


In particular, it holds that

$$
\begin{aligned}
& \frac{\partial}{\partial \alpha} \mathbb{E}_{\theta}[\Phi(X)]=\lim _{n \uparrow+\infty} \Xi_{n}^{(1)}(\Phi, \theta), \\
& \frac{\partial}{\partial \beta} \mathbb{E}_{\theta}[\Phi(X)]=\Xi^{(2)}(\Phi, \theta), \\
& \frac{\partial}{\partial \delta} \mathbb{E}_{\theta}[\Phi(X)]=\lim _{n \uparrow+\infty} \Xi_{n}^{(3)}(\Phi, \theta), \\
& \frac{\partial}{\partial \mu} \mathbb{E}_{\theta}[\Phi(X)]=\lim _{n \uparrow+\infty} \Xi_{n}^{(4)}(\Phi, \theta) .
\end{aligned}
$$

Proof. Throughout the proof, we write $\tilde{x}:=(x-\mu) / \alpha$. (Note that $\tilde{x}$ depends on the parameters $\alpha$ and $\mu$ although we suppress those for ease of notation.) The log-likelihood function is given by

$$
\ln f(x ; \theta)=2 \delta \ln (2 \cos (\beta / 2))-\ln (2 \pi \alpha)-\ln \Gamma(2 \delta)+\beta \widetilde{x}+\ln |\Gamma(\delta+i \widetilde{x})|^{2} .
$$

Recall that for $z \in \mathbb{C}$ with $\operatorname{Re}(z)>0$,

$$
|\Gamma(z)|^{2}=\Gamma(z) \overline{\Gamma(z)}=\Gamma(z) \Gamma(\bar{z})
$$

the definition of the digamma function

$$
\frac{\Gamma^{\prime}(z)}{\Gamma(z)}=-\frac{1}{z}-\gamma-\sum_{k=1}^{+\infty}\left(\frac{1}{k+z}-\frac{1}{k}\right)
$$

where $z \in \mathbb{C}$ with $\operatorname{Re}(z)>0$ and where $\gamma:=\lim _{n \uparrow+\infty}\left(\sum_{k=1}^{n} k^{-1}-\ln n\right) \approx 0.5772$ the Euler-Mascheroni constant, and that for each $a>0$ and $b \in \mathbb{R}$,

$$
\begin{aligned}
& \operatorname{Re}\left(\frac{\Gamma^{\prime}(a+i b)}{\Gamma(a+i b)}\right)=-\frac{a}{a^{2}+b^{2}}-\gamma-\sum_{k=1}^{+\infty}\left(\frac{k+a}{(k+a)^{2}+b^{2}}-\frac{1}{k}\right), \\
& \operatorname{Im}\left(\frac{\Gamma^{\prime}(a+i b)}{\Gamma(a+i b)}\right)=\sum_{k=0}^{+\infty} \frac{b}{(k+a)^{2}+b^{2}} .
\end{aligned}
$$

Since the density function $f(x ; \theta)$ takes only real values, it holds that for each $k=0,1,2$,

$$
\nabla_{\theta}^{k} \operatorname{Im}(\ln \Gamma(\delta+i \widetilde{x})+\ln \Gamma(\delta-i \widetilde{x}))=0,
$$

With the help of those properties, a tedious yet straightforward computation yields

$$
\nabla_{\theta}(\ln f(x ; \theta))=\left[\begin{array}{l}
-\frac{1}{\alpha}-\frac{\beta}{\alpha} \widetilde{x}+\frac{2}{\alpha} \widetilde{x}^{2} \sum_{k=0}^{+\infty} \frac{1}{(k+\delta)^{2}+\widetilde{x}^{2}} \\
\widetilde{x}-\delta \tan (\beta / 2) \\
2 \ln (2 \cos (\beta / 2))+2 \sum_{k=0}^{+\infty}\left(\frac{1}{k+2 \delta}-\frac{k+\delta}{(k+\delta)^{2}+\widetilde{x}^{2}}\right) \\
-\frac{\beta}{\alpha}+\frac{2 \widetilde{x}}{\alpha} \sum_{k=0}^{+\infty} \frac{1}{(k+\delta)^{2}+\widetilde{x}^{2}}
\end{array}\right] .
$$

Observe next that

$$
\begin{aligned}
\nabla_{\theta}\left(\mathbb{E}_{\theta}[\Phi(X)]\right) & =\nabla_{\theta}\left(\int_{\mathbb{R}} \Phi(x) f(x ; \theta) d x\right) \\
& =\int_{\mathbb{R}} \Phi(x) \nabla_{\theta}(\ln f(x ; \theta)) f(x ; \theta) d x \\
& =\mathbb{E}_{\theta}\left[\Phi(X) \nabla_{\theta}(\ln f(X ; \theta))\right],
\end{aligned}
$$

where the second equality holds by the fact that $f(x ; \theta)>0$ over $\mathbb{R}$ and the interchange of the derivative and the integration can be justified as follows. It holds by the Taylor theorem that for $h \in \mathbb{R}^{4}$ such that $\|h\|=1$ and a fixed constant $\varepsilon>0$ 
satisfying $\theta+\varepsilon h \in \Theta$,

$$
\begin{aligned}
\mid \int_{\mathbb{R}} \Phi & (x) \frac{\ln f(x ; \theta+\varepsilon h)-\ln f(x ; \theta)}{\varepsilon} f(x ; \theta) d x \mid \\
& =\left|\int_{\mathbb{R}} \Phi(x)\left\langle h,\left.\int_{0}^{1} \nabla_{\lambda}(\ln f(x ; \lambda))\right|_{\lambda=\theta+s \varepsilon h} d s\right\rangle f(x ; \theta) d x\right| \\
& \leq \int_{\mathbb{R}} \Phi(x) \sup _{s \in[0,1]}\left\|\left.\nabla_{\lambda}(\ln f(x ; \lambda))\right|_{\lambda=\theta+s \varepsilon h}\right\| f(x ; \theta) d x<+\infty,
\end{aligned}
$$

where the last inequality holds by the condition (3.1) and the linear growth of the supremum in variable $x$. This justifies the interchange by the dominated convergence theorem.

Finally, upper and lower bounds for the infinite sums in (3.6) can be derived by an elementary calculus as follows. Observe that for each $x \in \mathbb{R}$ and $n \in \mathbb{N}$,

$$
\int_{n}^{+\infty} \frac{1}{(y+\delta)^{2}+x^{2}} d y<\sum_{k=n}^{+\infty} \frac{1}{(k+\delta)^{2}+x^{2}}<\frac{1}{(n+\delta)^{2}+x^{2}}+\int_{n+1}^{+\infty} \frac{1}{(y-1+\delta)^{2}+x^{2}} d y .
$$

By evaluating the integral, we get

$$
0<\sum_{k=n}^{+\infty} \frac{1}{(k+\delta)^{2}+x^{2}}-\frac{1}{|x|}\left(\frac{\pi}{2}-\tan ^{-1}\left(\frac{n+\delta}{|x|}\right)\right)<\frac{1}{(n+\delta)^{2}+x^{2}} .
$$

In a similar manner, we get

$$
-\frac{\delta}{(n+2 \delta)(n+\delta)}<\sum_{k=n}^{+\infty}\left(\frac{1}{k+2 \delta}-\frac{k+\delta}{(k+\delta)^{2}+x^{2}}\right)-\frac{1}{2} \ln \frac{(n+\delta)^{2}+x^{2}}{(n+2 \delta)^{2}}<\frac{x^{2}}{(n+\delta)\left((n+\delta)^{2}+x^{2}\right)} .
$$

Note that for each $n \in \mathbb{N}$, all the bounds are uniformly bounded in $x$ over $\mathbb{R}$. The proof is complete.

If the function $\Phi$ is non-negative, more precise bias bounds can be derived.

Corollary 3.2. Under the setting of Theorem 3.1 if $\Phi$ is non-negative, then it holds that for each $n \in \mathbb{N}$,

$$
\begin{gathered}
0<\frac{\partial}{\partial \alpha} \mathbb{E}_{\theta}[\Phi(X)]-\Xi_{n}^{(1)}(\Phi, \theta)<\frac{2}{\alpha} \mathbb{E}_{\theta}\left[\Phi(X) \frac{\widetilde{X}^{2}}{(n+\delta)^{2}+\widetilde{X}^{2}}\right], \\
\frac{-2 \delta}{(n+2 \delta)(n+\delta)} \mathbb{E}_{\theta}[\Phi(X)]<\frac{\partial}{\partial \delta} \mathbb{E}_{\theta}[\Phi(X)]-\Xi_{n}^{(3)}(\Phi, \theta)<\frac{2}{n+\delta} \mathbb{E}_{\theta}\left[\Phi(X) \frac{\widetilde{X}^{2}}{(n+\delta)^{2}+\widetilde{X}^{2}}\right], \\
\frac{2}{\alpha} \mathbb{E}_{\theta}\left[\Phi(X) \frac{\widetilde{X} \mathbb{1}(\widetilde{X}<0)}{(n+\delta)^{2}+\widetilde{X}^{2}}\right]<\frac{\partial}{\partial \mu} \mathbb{E}_{\theta}[\Phi(X)]-\Xi_{n}^{(4)}(\Phi, \theta)<\frac{2}{\alpha} \mathbb{E}_{\theta}\left[\Phi(X) \frac{\widetilde{X} \mathbb{1}(\widetilde{X}>0)}{(n+\delta)^{2}+\widetilde{X}^{2}}\right] .
\end{gathered}
$$

The above formulas can directly be applied to the so-called Greeks in mathematical finance. Let $\left\{X_{t}: t \geq 0\right\}$ be a Meixner process such that $\mathscr{L}\left(X_{1}\right)=\operatorname{Meixner}(\alpha, \beta, \delta, \mu)$. Fix $S_{0}>0, r \geq 0, \sigma>0$ and $T>0$. Define an asset price dynamics $\left\{S_{t}: t \geq 0\right\}$ driven by this Meixner process by

$$
S_{t}:=S_{0} e^{r t} \frac{e^{\sigma X_{t}}}{\mathbb{E}\left[e^{\sigma X_{t}}\right]},
$$

with $\left\{e^{-r t} S_{t}: t \geq 0\right\}$ being a martingale with respect to the natural filtration generated by $\left\{X_{t}: t \geq 0\right\}$. In mathematical finance, the sensitivity indices below are called delta, vega, theta and rho, respectively.

Corollary 3.3. Let $Z$ be a random variable with $\operatorname{Meixner}\left(\theta_{1}, \theta_{2}, \theta_{3}, \theta_{4}\right)$ such that

$$
\left[\theta_{1}, \theta_{2}, \theta_{3}, \theta_{4}\right]:=\left[\sigma \alpha, \beta, T \delta, \ln S_{0}+r T-\ln \left(\frac{\cos (\beta / 2)}{\cos ((\sigma \alpha-\beta) / 2)}\right)^{2 \delta T}\right] .
$$


Let $\widetilde{\Phi}(z):=\Phi\left(e^{z}\right)$, where $\Phi$ is a measurable function from $\mathbb{R}$ to $\mathbb{R}$ such that $\mathbb{E}_{\theta}[|\widetilde{\Phi}(Z)|(1 \vee|Z|)]<+\infty$. It then holds that

$$
\begin{aligned}
\frac{\partial}{\partial S_{0}} \mathbb{E}\left[e^{-r T} \Phi\left(S_{T}\right)\right] & =\frac{e^{-r T}}{S_{0}} \lim _{n \uparrow+\infty} \Xi_{n}^{(4)}(\widetilde{\Phi} ; \theta) \\
\frac{\partial}{\partial \sigma} \mathbb{E}\left[e^{-r T} \Phi\left(S_{T}\right)\right]= & e^{-r T} \alpha \lim _{n \uparrow+\infty} \Xi_{n}^{(1)}(\widetilde{\Phi} ; \theta)-S_{0} \alpha \delta T \tan \frac{\sigma \alpha-\beta}{2} \frac{\partial}{\partial S_{0}} \mathbb{E}_{\theta}\left[e^{-r T} \Phi\left(S_{T}\right)\right], \\
\frac{\partial}{\partial T} \mathbb{E}\left[e^{-r T} \Phi\left(S_{T}\right)\right]= & -r \mathbb{E}_{\theta}\left[e^{-r T} \Phi\left(S_{T}\right)\right]+e^{-r T} \delta \lim _{n \uparrow+\infty} \Xi_{n}^{(3)}(\widetilde{\Phi} ; \theta) \\
& +\frac{\theta_{4}-\ln S_{0}}{T} S_{0} \frac{\partial}{\partial S_{0}} \mathbb{E}_{\theta}\left[e^{-r T} \Phi\left(S_{T}\right)\right] \\
\frac{\partial}{\partial r} \mathbb{E}\left[e^{-r T} \Phi\left(S_{T}\right)\right]= & -T \mathbb{E}_{\theta}\left[e^{-r T} \Phi\left(S_{T}\right)\right]+T S_{0} \frac{\partial}{\partial S_{0}} \mathbb{E}_{\theta}\left[e^{-r T} \Phi\left(S_{T}\right)\right] .
\end{aligned}
$$

Proof. With the help of the relation (2.4), we get

$$
\ln S_{T}=\sigma X_{T}+\ln S_{0}+T\left(r-\sigma \mu-\ln \left(\frac{\cos (\beta / 2)}{\cos ((\sigma \alpha-\beta) / 2)}\right)\right)^{2 \delta} \stackrel{\mathscr{L}}{=} Z,
$$

where the random variable $Z$ is defined as before. The rest is straightforward.

The second-order parameter sensitivities can be investigated based on

$$
\begin{aligned}
\operatorname{Hess}_{\theta}\left(\mathbb{E}_{\theta}[\Phi(X)]\right) & =\nabla_{\theta}^{\top}\left(\int_{\mathbb{R}} \Phi(x) \nabla_{\theta} f(x ; \theta) d x\right) \\
& =\int_{\mathbb{R}} \Phi(x) \operatorname{Hess}_{\theta}(f(x ; \theta)) d x \\
& =\int_{\mathbb{R}} \Phi(x)\left[\operatorname{Hess}_{\theta}(\ln f(x ; \theta))+\left(\nabla_{\theta}(\ln f(x ; \theta))\right)\left(\nabla_{\theta}(\ln f(x ; \theta))\right)^{\top}\right] f(x ; \theta) d x \\
& =\mathbb{E}_{\theta}\left[\Phi(X)\left(\operatorname{Hess}_{\theta}(\ln f(X ; \theta))+\left(\nabla_{\theta}(\ln f(X ; \theta))\right)\left(\nabla_{\theta}(\ln f(X ; \theta))\right)^{\top}\right)\right],
\end{aligned}
$$

where the gradient is given in (3.6). Note that we here need $\mathbb{E}_{\theta}\left[|\Phi(X)|\left(1 \vee|X|^{2}\right)\right]<+\infty$, rather than (3.1). The diagonal entries of the Hessian matrix are given by

$$
\begin{aligned}
& \operatorname{Hess}_{\theta}(\ln f(x ; \theta))_{1,1}=\frac{1+2 \beta \widetilde{x}}{\alpha^{2}}+\frac{2 \widetilde{x}^{2}}{\alpha^{2}} \sum_{k=0}^{+\infty} \frac{1}{(k+\delta)^{2}+\widetilde{x}^{2}}-\frac{4 \widetilde{x}^{4}}{\alpha^{2}} \sum_{k=0}^{+\infty} \frac{1}{\left((k+\delta)^{2}+\widetilde{x}^{2}\right)^{2}}, \\
& \operatorname{Hess}_{\theta}(\ln f(x ; \theta))_{2,2}=-\frac{\delta}{2 \cos ^{2}(\beta / 2)}, \\
& \operatorname{Hess}_{\theta}(\ln f(x ; \theta))_{3,3}=-4 \sum_{k=0}^{+\infty} \frac{1}{(k+2 \delta)^{2}}+2 \sum_{k=0}^{+\infty} \frac{1}{(k+\delta)^{2}+\widetilde{x}^{2}}-4 \widetilde{x}^{2} \sum_{k=0}^{+\infty} \frac{1}{\left((k+\delta)^{2}+\widetilde{x}^{2}\right)^{2}}, \\
& \operatorname{Hess}_{\theta}(\ln f(x ; \theta))_{4,4}=-\frac{2}{\alpha^{2}} \sum_{k=0}^{+\infty} \frac{1}{(k+\delta)^{2}+\widetilde{x}^{2}}+\frac{4 \widetilde{x}^{2}}{\alpha^{2}} \sum_{k=0}^{+\infty} \frac{1}{\left((k+\delta)^{2}+\widetilde{x}^{2}\right)^{2}},
\end{aligned}
$$

while the off-diagonal entries are given by

$$
\begin{aligned}
& \operatorname{Hess}_{\theta}(\ln f(x ; \theta))_{1,2}=-\frac{\widetilde{x}}{\alpha}, \quad \operatorname{Hess}_{\theta}(\ln f(x ; \theta))_{2,3}=-\tan \frac{\beta}{2}, \quad \operatorname{Hess}_{\theta}(\ln f(x ; \theta))_{2,4}=-\frac{1}{\alpha}, \\
& \operatorname{Hess}_{\theta}(\ln f(x ; \theta))_{1,3}=-\frac{4 \widetilde{x}^{2}}{\alpha} \sum_{k=0}^{+\infty} \frac{k+\delta}{\left((k+\delta)^{2}+\widetilde{x}^{2}\right)^{2}}, \\
& \operatorname{Hess}_{\theta}(\ln f(x ; \theta))_{1,4}=\frac{\beta}{\alpha^{2}}-\frac{4 \widetilde{x}}{\alpha^{2}} \sum_{k=0}^{+\infty} \frac{1}{(k+\delta)^{2}+\widetilde{x}^{2}}+\frac{4 \widetilde{x}^{3}}{\alpha^{2}} \sum_{k=0}^{+\infty} \frac{1}{\left((k+\delta)^{2}+\widetilde{x}^{2}\right)^{2}}, \\
& \operatorname{Hess}_{\theta}(\ln f(x ; \theta))_{3,4}=-\frac{4 \widetilde{x}}{\alpha} \sum_{k=0}^{+\infty} \frac{k+\delta}{\left((k+\delta)^{2}+\widetilde{x}^{2}\right)^{2}} .
\end{aligned}
$$


We do not state unbiased estimators, such as (3.2)-(3.5), to avoid overloading this section with nonessential lengthy formulas, while the derivation is straightforward with (3.6), (3.7), 3.10), and the following approximations; for each $x \in \mathbb{R}$,

$$
\begin{gathered}
0<\sum_{k=n}^{+\infty} \frac{1}{\left((k+\delta)^{2}+x^{2}\right)^{2}}-\frac{1}{2 x^{2}}\left(\frac{1}{|x|}\left(\frac{\pi}{2}-\tan ^{-1} \frac{n+\delta}{|x|}\right)-\frac{n+\delta}{(n+\delta)^{2}+x^{2}}\right)<\frac{1}{\left((n+\delta)^{2}+x^{2}\right)^{2}}, \\
0<\sum_{k=n}^{+\infty} \frac{k+\delta}{\left((k+\delta)^{2}+x^{2}\right)^{2}}-\frac{1}{2} \frac{1}{(n+\delta)^{2}+x^{2}}<\frac{n+\delta}{\left((n+\delta)^{2}+x^{2}\right)^{2}} .
\end{gathered}
$$

In the context of financial Greeks, the second derivatives $\partial_{\mu}^{2}, \partial_{\mu} \partial_{\alpha}$ and $\partial_{\alpha}^{2}$ can be associated with gamma, vanna and volga, respectively.

\subsection{A Simple Simulation Method}

Given estimation formulas, such as (3.2)-3.5), it remains to generate a sequence of iid random variables with common Meixner $\alpha, \beta, \delta, \mu)$ distribution. The most straightforward approach seems to be the inversion method with the use of the explicit density function (2.2). (See, for example, Imai and Tan [10] for a numerical inversion method of the Meixner distribution in connection with the quasi-Monte Carlo method.) An exact simulation method based on acceptance-rejection sampling is proposed in Grigoletto and Privasi [8]. This acceptance-rejection sampling method employs the Johnson translation system and requires some amount of initial numerical computations. Apart from general parameter settings, it holds that when $(\alpha, \delta)=(1,1 / 2)$ is fixed,

$$
\mathscr{L}\left(\frac{1}{2 \pi} \ln \frac{G_{1}}{G_{2}}\right)=\operatorname{Meixner}(1, \beta, 1 / 2,0) .
$$

This identity in law is very useful for simulation, where $G_{1}$ and $G_{2}$ are independent gamma random variables, respectively, with parameters $(1 / 2+\beta /(2 \pi), 1)$ and $(1 / 2-\beta /(2 \pi), 1)$. (See Yano et al. [20].) Here, fixing $\delta=1 / 2$ is way too restrictive since in principle, this parameter corresponds to the time of the associated Lévy process. (In contrast, fixing $\alpha=1$ is not at all, due to the scaling property (2.4.)

In this subsection, we propose a simple exact simulation method for arbitrary Meixner distributions. Our method consists of two components of acceptance-rejection sampling and the Esscher density transform. Let us begin with the acceptance-rejection sampling method. It holds by the well known result of Devroye [1] that for $x \in \mathbb{R}$,

$$
f(x ; \theta) \leq \min \left[C_{1}(\theta), \frac{\left.C_{2}(\theta)\right)}{x^{2}}\right]:=q(x ; \theta),
$$

where

and that

$$
C_{1}(\theta):=\frac{1}{2 \pi} \int_{\mathbb{R}}|\varphi(y ; \theta)| d y, \quad C_{2}(\theta):=\frac{1}{2 \pi} \int_{\mathbb{R}}\left|\varphi^{\prime \prime}(y ; \theta)\right| d y,
$$

$$
C_{3}(\theta):=\int_{\mathbb{R}} q(x ; \theta) d x=4 \sqrt{C_{1}(\theta) C_{2}(\theta)} .
$$

Let $U_{1}$ and $U_{2}$ be iid uniform random variables on $(-1,+1)$ and define $V:=\sqrt{C_{2}(\theta) / C_{1}(\theta)} U_{1} / U_{2}$. The random variable $V$ admits a probability density $\left(C_{3}(\theta)\right)^{-1} q(x ; \theta)$. Based on the above facts, we can employ a simulation method based on acceptance-rejection sampling as follows.

\section{Algorithm 1;}

Step 1. Generate $V$ as above and $U$ as $U(0,1)$ independent of $V$. If $|V|<\sqrt{C_{2}(\theta) / C_{1}(\theta)}$, then go to Step 3 .

Step 2. If $C_{2}(\theta) U<f(V ; \theta) V^{2}$, then exit with $X(\theta) \leftarrow V$. Otherwise, go to Step 1 .

Step 3. If $C_{1}(\theta) U<f(V ; \theta)$, then exit with $X(\theta) \leftarrow V$. Otherwise, go to Step 1 .

This is an exact simulation algorithm, that is, $\mathscr{L}(X(\theta))=\operatorname{Meixner}(\alpha, \beta, \delta, \mu)$. The acceptance rate at Step 1 of Algorithm 1 is $C_{3}(\theta)^{-1}$, while the expected number of times Step 1 is executed is thus $C_{3}(\theta)$. (See Kawai and Masuda [16] for an application of this algorithm to the tempered stable distribution.) It however seems difficult to find the quantities $C_{1}(\theta)$ and $C_{2}(\theta)$ in closed form for arbitrary $\theta$. The simulation method we propose avoids the initial numerical integration for the integrals (3.13). In what follows, we fix $\alpha$ and $\delta$ arbitrarily, and use the notation $\theta_{(\beta, \mu)}:=(\alpha, \beta, \delta, \mu)$ for convenience. The following results play an essential role. In short, when the distribution is symmetric, that is $\beta=0$, the above constants are more tractable. 
Lemma 3.4. It holds that

$$
\begin{gathered}
C_{1}\left(\theta_{(0, \mu)}\right)=\frac{2^{2 \delta-1} \Gamma(\delta)^{2}}{\pi \alpha \Gamma(2 \delta)}, \quad \mu \in \mathbb{R} \\
C_{2}\left(\theta_{(0,0)}\right)<\frac{\alpha^{2} \delta}{2} \max (1,2 \delta) C_{1}\left(\theta_{(0,0)}\right)=: C_{4}\left(\theta_{(0,0)}\right) .
\end{gathered}
$$

Proof. The first claim holds by

$$
\frac{1}{2 \pi} \int_{\mathbb{R}}\left|\varphi\left(y ; \theta_{(0, \mu)}\right)\right| d y=\frac{1}{2 \pi} \int_{\mathbb{R}} \varphi\left(y ; \theta_{(0,0)}\right) d y=\frac{1}{2 \pi} \int_{\mathbb{R}} e^{-i 0 y} \varphi\left(y ; \theta_{(0,0)}\right) d y=f\left(0 ; \theta_{(0,0)}\right) .
$$

Next, observe that

$$
\varphi^{\prime \prime}\left(y ; \theta_{(0,0)}\right)=\frac{\alpha^{2} \delta}{2} \varphi\left(y ; \theta_{(0,0)}\right)\left((1+2 \delta) \tanh ^{2}\left(\frac{\alpha}{2} y\right)-1\right)
$$

and

$$
\left|(1+2 \delta) \tanh ^{2}\left(\frac{\alpha}{2} y\right)-1\right| \leq \max (1,2 \delta), \quad y \in \mathbb{R} .
$$

The second claim holds by combining those with the first claim.

Using those results, the algorithm is reformulated as follows. Observe that

$$
\begin{gathered}
f\left(x ; \theta_{(0,0)}\right) \leq q\left(x ; \theta_{(0,0)}\right) \leq \min \left[C_{1}\left(\theta_{(0,0)}\right), \frac{C_{4}\left(\theta_{(0,0)}\right)}{x^{2}}\right]=: p\left(x ; \theta_{(0,0)}\right), \quad x \in \mathbb{R}, \\
C_{5}\left(\theta_{(0,0)}\right):=\int_{\mathbb{R}} p\left(x ; \theta_{(0,0)}\right) d x=4 \sqrt{C_{1}\left(\theta_{(0,0)}\right) C_{4}\left(\theta_{(0,0)}\right)}=\frac{2^{2 \delta} \Gamma(\delta)^{2}}{\pi \Gamma(2 \delta)} \max (\sqrt{2 \delta}, 2 \delta),
\end{gathered}
$$

where $q\left(x ; \theta_{(0,0)}\right)$ is defined in (3.12). As for Algorithm 1, the quantity $C_{5}\left(\theta_{(0,0)}\right)^{-1}$ acts as the acceptance rate of our algorithm. Note that the constant $C_{5}\left(\theta_{(0,0)}\right)$ depends only on $\delta$. Define

$$
V:=\sqrt{\frac{C_{4}\left(\theta_{(0,0)}\right)}{C_{1}\left(\theta_{(0,0)}\right)}} \frac{U_{1}}{U_{2}}=\frac{\alpha}{2} \max (\sqrt{2 \delta}, 2 \delta) \frac{U_{1}}{U_{2}}
$$

where $U_{1}$ and $U_{2}$ are again independent uniform random variables on $(-1,+1)$. The algorithm is as follows.

\section{Algorithm 2;}

Step 1. Generate $V$ as $(3.15)$ and $U$ as $U(0,1)$ independent of $V$. If $|V|<\alpha \max (\sqrt{2 \delta}, 2 \delta) / 2$, then go to Step 3 .

Step 2. If $C_{4}\left(\theta_{(0,0)}\right) U<f\left(V ; \theta_{(0,0)}\right) V^{2}$, that is,

$$
\frac{\max (1,2 \delta) \alpha^{2} \Gamma(\delta+1)^{2}}{2 \delta} U<\left|\Gamma\left(\delta+i \frac{V}{\alpha}\right)\right|^{2} V^{2}
$$

then exit with $X\left(\theta_{(0,0)}\right) \leftarrow V$. Otherwise, go to Step 1 .

Step 3. If $C_{1}\left(\theta_{(0,0)}\right) U<f\left(V ; \theta_{(0,0)}\right)$, that is,

$$
\Gamma(\delta)^{2} U<\left|\Gamma\left(\delta+i \frac{V}{\alpha}\right)\right|^{2}
$$

then exit with $X\left(\theta_{(0,0)}\right) \leftarrow V$. Otherwise, go to Step 1 .

The acceptance rate at Step 1 of Algorithm 2 is given by $C_{5}\left(\theta_{(0,0)}\right)^{-1}$ and thus the expected number of times Step 1 is executed is $C_{5}\left(\theta_{(0,0)}\right)$. It is discussed in [1] that the quantity $C_{2}(\theta)$ in $(3.13)$ is minimized when the random variable to be simulated is set centered. In fact, this applies implicitly to our case $(\beta=0)$ as well; the upper bound given in (3.14) is minimal with respect to $\mu$. Clearly, fixing $\mu=0$ is not a restriction at all as the original location $\mu$ can easily be summed back afterwards. To illustrate how Algorithm 2 works, we provide Figure 1 The key quantity is the acceptance rate $C_{5}\left(\theta_{(0,0)}\right)^{-1}$ illustrated in the left figure. The acceptance rate takes the global maxima of 2 at $\delta=1 / 2$, while it tends monotonically to 0 as either $\delta \downarrow 0$ or $\delta \uparrow+\infty$. (Recall that this acceptance-rejection sampling method is not quite of use 


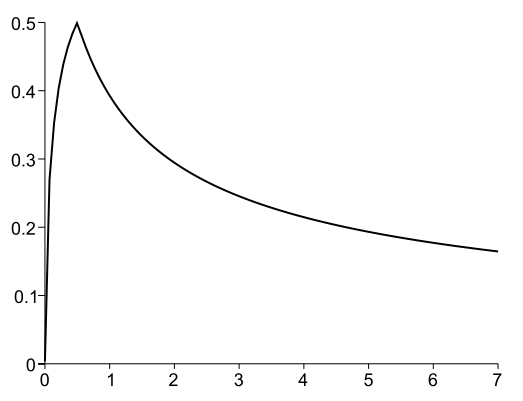

$(x, y)=\left(\delta, C_{5}\left(\theta_{(0,0)}\right)^{-1}\right)$

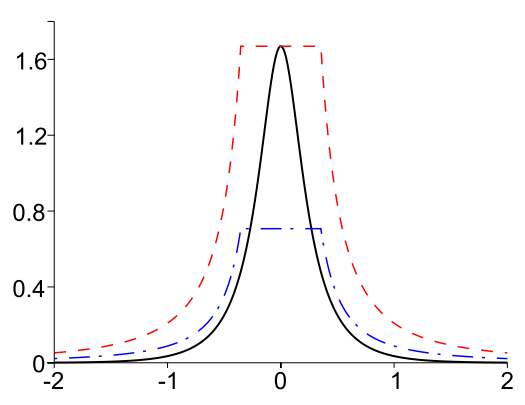

$\left(\delta, C_{5}\left(\theta_{(0,0)}\right)^{-1}\right)=(0.25,0.4236)$

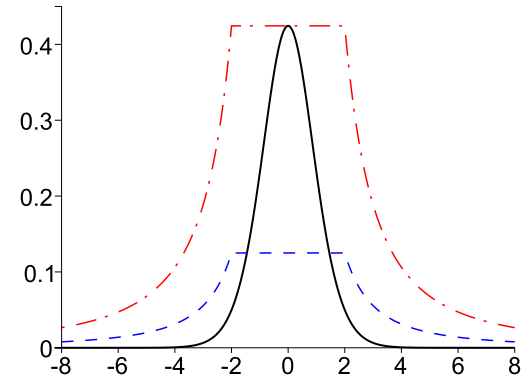

$\left(\delta, C_{5}\left(\theta_{(0,0)}\right)^{-1}\right)=(2.0,0.2945)$

Figure 1: The acceptance rate $C_{5}\left(\theta_{(0,0)}\right)^{-1}$ of acceptance-rejection sampling (left). Comparisons (center and right) of true density function $f\left(x ; \theta_{(0,0)}\right)(-)$, proposal density function $C_{5}\left(\theta_{(0,0)}\right)^{-1} p\left(x ; \theta_{(0,0)}\right)(-\cdot-)$, and bounding function $p\left(x ; \theta_{(0,0)}\right)$ (- - ), where $\alpha=1$.

when $\delta=1 / 2$ due to the convenient identity (3.11).) Next, as drawn in the center and the right, the distance between the true $f\left(x ; \theta_{(0,0)}\right)$ and the (non-standardized) proposal $p\left(x ; \theta_{(0,0)}\right)$ roughly tells the efficiency of the algorithm. Loosely speaking, the algorithm is efficient when those two are close.

Next, we add asymmetry to the Meixner distribution through the Esscher transform. Due to Algorithm 2, a sequence of iid random variables with common $\operatorname{Meixner}(\alpha, 0, \delta, \mu)$ distribution is now available. We next transform Meixner $(\alpha, 0, \delta, \mu)$ into Meixner $(\alpha, \beta, \delta, \mu)$ with an arbitrary $\beta$ such that $|\beta|<\pi$ for Monte Carlo estimation purposes. Let $\mathscr{F}$ be the minimal $\sigma$-field generated by the random variable $X$ and let $\mathbb{P} \mid \mathscr{F}$ denote the restriction of the probability measure $\mathbb{P}$ to $\mathscr{F}$. It is known that the probability measures $\mathbb{P}_{\theta_{(\beta, \mu)}}$ and $\mathbb{P}_{\theta_{(0, \mu)}}$ are equivalent with

$$
\left.\frac{d \mathbb{P}_{\theta_{(\beta, \mu)}}}{d \mathbb{P}_{\theta_{(0, \mu)}}}\right|_{\mathscr{F}}=\frac{e^{\beta X / \alpha}}{\mathbb{E}_{\theta_{(0, \mu)}}\left[e^{\beta X / \alpha}\right]}=\exp \left[\frac{\beta}{\alpha}(X-\mu)+2 \delta \ln \cos \frac{\beta}{2}\right], \quad \mathbb{P}_{\theta_{(0, \mu)}} \text {-a.s. }
$$

(See, for example, Grigelionis [7].) With this Radon-Nykodym derivative, it holds that for each measurable function $\Psi$ mapping from $\mathbb{R}$ to $\mathbb{R}$ such that $\mathbb{E}_{\theta_{(\beta, \mu)}}[|\Psi(X)|]<+\infty$,

$$
\mathbb{E}_{\theta_{(\beta, \mu)}}[\Psi(X)]=\mathbb{E}_{\theta_{(0, \mu)}}\left[\left.\frac{d \mathbb{P}_{\theta_{(\beta, \mu)}}}{d \mathbb{P}_{\theta_{(0, \mu)}}}\right|_{\mathscr{F}} \Psi(X)\right]=\mathbb{E}_{\theta_{(0,0)}}\left[\left.\frac{d \mathbb{P}_{\theta_{(\beta, 0)}}}{d \mathbb{P}_{\theta_{(0,0)}}}\right|_{\mathscr{F}} \Psi(X+\mu)\right]
$$

It is worth rephrasing that the (symmetric) Meixner $(\alpha, 0, \delta, \mu)$ distribution can be simulated exactly through Algorithm 2 for sample path generation purposes, while the the general (asymmetric) Meixner $(\alpha, \beta, \delta, \mu)$ distribution can be simulated for Monte Carlo estimation purposes only (in other words, only for computation of expectations) through our Esscher transform method after its symmetric and centered version Meixner $(\alpha, 0, \delta, 0)$ is simulated through Algorithm 2 .

\section{Numerical Illustrations}

We provide illustrative numerical results to support our theoretical analysis. Rather than presenting exhaustive Monte Carlo experiments, we first focus on estimation of the delta given in (3.9) with an indicator function $\Phi(x)=\mathbb{1}\left(e^{x}>K\right)$ for some $K>0$, which corresponds to a digital option in the context of mathematical finance. We take this example first for more precise comparison purpose, as the sensitivity with respect to the initial state $S_{0}$ reduces to the closed form

$$
\frac{\partial}{\partial S_{0}} \mathbb{E}_{\theta}\left[e^{-r T} \mathbb{1}\left(S_{T}>K\right)\right]=\frac{e^{-r T}}{S_{0}} f(\ln K ; \theta)
$$

where $\theta=\left[\theta_{1}, \theta_{2}, \theta_{3}, \theta_{4}\right]^{\top}$ is given by (3.8). In contrast, we have obtained the estimation formula

$$
\frac{\partial}{\partial S_{0}} \mathbb{E}_{\theta}\left[e^{-r T} \mathbb{1}\left(S_{T}>K\right)\right]=\lim _{n \uparrow+\infty} \frac{e^{-r T}}{S_{0}} \Xi_{n}^{(4)}\left(\mathbb{1}\left(e^{x}>K\right) ; \theta\right),
$$


due to Theorem 3.1 (i). Since $\Phi(x)=\mathbb{1}\left(e^{x}>K\right)$ is non-negative, we can employ the bias bounds given in Corollary 3.2 that is,

$$
\begin{array}{r}
C_{n}^{-}:=\frac{2 e^{-r T}}{\theta_{1} S_{0}} \mathbb{E}_{\theta}\left[\mathbb{1}\left(S_{T}>K\right) \frac{\left(X-\theta_{4}\right) / \theta_{1} \mathbb{1}\left(X<\theta_{4}\right)}{\left(n+\theta_{3}\right)^{2}+\left(\left(X-\theta_{4}\right) / \theta_{1}\right)^{2}}\right] \\
\leq \frac{e^{-r T}}{S_{0}} f(\ln K ; \theta)
\end{array}
$$

In other words, it holds that

$$
\frac{e^{-r T}}{S_{0}} f(\ln K ; \theta)-C_{n}^{+} \leq \frac{e^{-r T}}{S_{0}} \Xi_{n}^{(4)}\left(\mathbb{1}\left(e^{x}>K\right) ; \theta\right) \leq \frac{e^{-r T}}{S_{0}} f(\ln K ; \theta)-C_{n}^{-} .
$$

Note that $C_{n}^{+}>0, C_{n}^{-}<0$ and $\lim _{n \uparrow+\infty} C_{n}^{+}=\lim _{n \uparrow+\infty} C_{n}^{-}=0$. Throughout this section, we fix the model parameters $(\alpha, \beta, \delta)=(0.3977,-1.4940,0.3462)$ and $\left(\sigma, r, T, S_{0}, K\right)=(1,0.05,1.0,100,85)$. We draw in Figure 3 Monte Carlo convergence in estimation of $\frac{e^{-r T}}{S_{0}} \Xi_{n}^{(4)}\left(\mathbb{1}\left(e^{x}>K\right) ; \theta\right)$ with $n=3,5,10$. The quantities $C_{n}^{+}$and $C_{n}^{-}$are estimated by Monte Carlo simulation as well. All the Monte Carlo simulations are performed with Algorithm 2 and the Esscher transform (3.16). It can be observed that as $n$ increases, the limiting value $\frac{e^{-r T}}{S_{0}} \Xi_{n}^{(4)}\left(\mathbb{1}\left(e^{x}>K\right) ; \theta\right)$ approaches to the true value $\frac{e^{-r T}}{S_{0}} f(\ln K ; \theta)$. As is often the case, the larger truncation $n$ requires a more amount of computation in return for a smaller bias. It seems that the theoretical bounds are sufficiently tight at early stage of the truncation, where computational load is still reasonable.
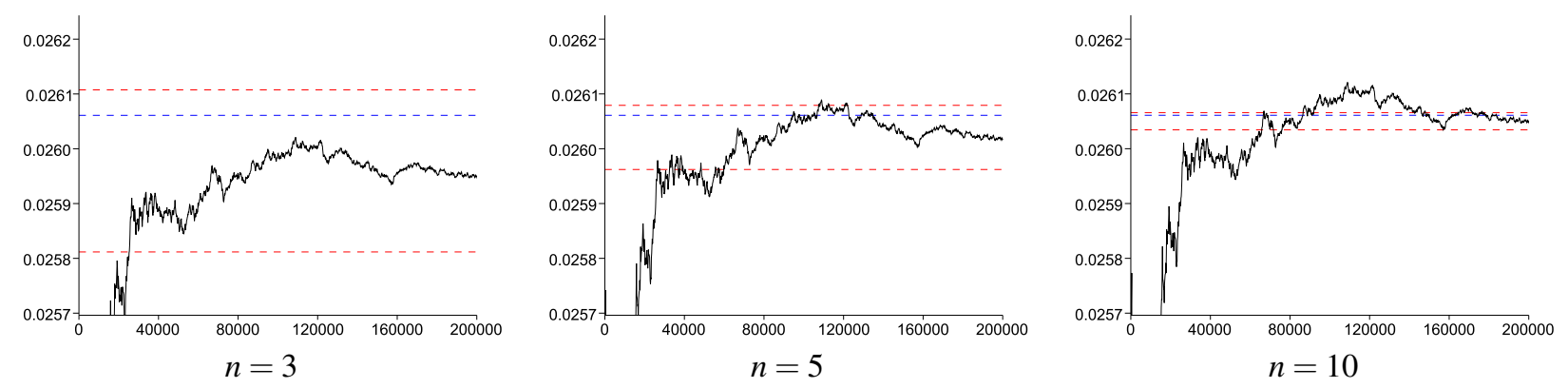

Figure 2: Monte Carlo convergence in estimation of $\frac{e^{-r T}}{S_{0}} \Xi_{n}^{(4)}\left(\mathbb{1}\left(e^{x}>K\right) ; \theta\right)$ with $n=3,5,10$. Three horizontal lines indicate the true value $\frac{e^{-r T}}{S_{0}} f(\ln K ; \theta)$ and its theoretical bounds $\frac{e^{-r T}}{S_{0}} f(\ln K ; \theta)-C_{n}^{ \pm}$.

Next, we test the formulas in Corollary 3.3 for the standard European call option, more relevant in financial applications, under the same parameter setting. (We do not consider the sensitivity $(\partial / \partial r)$ with respect to the risk-free rate, as it does not involve any of our results.) In our formulation, this corresponds to $\Phi(x)=\max \left(e^{x}-K, 0\right)=:\left(e^{x}-K\right)_{+} \cdot$ Observe that

$$
\left|\Phi\left(e^{x}\right)\right|(1 \vee|x|) f(x ; \theta) \sim \begin{cases}x^{2 \delta} \exp \left[\left(1-\frac{\pi+\beta}{\alpha}\right) x\right], & \text { as } x \uparrow+\infty, \\ 0, & \text { as } x \downarrow-\infty,\end{cases}
$$

where we have used the semi-heavy tails (2.3). Under our parameter setting, those tails are obviously integrable. Unlike the case of the digital option, it is difficult to derive explicit formulas for the sensitivities here. We thus estimate the true value through the standard central finite difference method, for instance,

$$
\frac{\partial}{\partial S_{0}} \mathbb{E}_{\theta}\left[e^{-r T}\left(S_{T}-K\right)_{+}\right] \approx \frac{\mathbb{E}_{\theta}\left[e^{-r T}\left((1+\varepsilon) S_{T}-K\right)_{+}\right]-\mathbb{E}_{\theta}\left[e^{-r T}\left((1-\varepsilon) S_{T}-K\right)_{+}\right]}{2 \varepsilon S_{0}}
$$

with some small positive $\varepsilon$. As the max function has a non-differentiable point and the perturbation $\varepsilon$ is strictly positive, the above right hand side is an approximation (with some bias) of the left hand side. Here, we fix $\varepsilon=0.001$. As earlier, 
the payoff function $\Phi(x)$ is non-negative, we employ the bias bounds of Corollary 3.2. (In these instances, the upper bounds are very close to, or even exactly same as, the corresponding true values.) Let us emphasize that our formula for $(\partial / \partial T)$ is of particular interest, as the finite difference method for theta $(\partial / \partial T)$ is significantly expensive in the sense that the two random variables $X_{T+\varepsilon}$ and $X_{T-\varepsilon}$ have to be generated separately, while the parameters $S_{0}$ and $\sigma$ are purely exogenous.
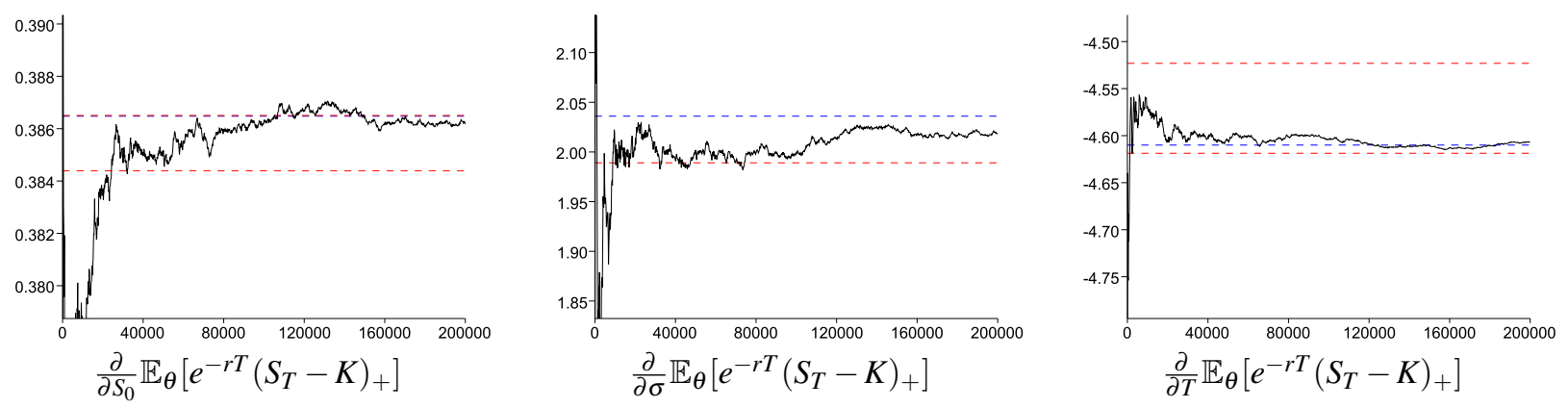

Figure 3: Monte Carlo convergence in estimation of delta $\left(\partial / \partial S_{0}\right)$, vega $(\partial / \partial \sigma)$ and theta $(\partial / \partial T)$ for the standard European call option. Horizontal lines indicate the true value (blue) and bounds (red).

\section{References}

[1] Devroye, L. (1981) On the computer generation of random variables with a given characteristic function, Computers and Mathematics with Applications, 7(6) 547-552.

[2] El-Khatib, Y., Privault, N. (2004) Computations of Greeks in a market with jumps via the Malliavin calculus, Finance and Stochastics, 8(2) 161-179.

[3] Fournié, E., Lasry, J. M., Lebuchoux, J., Lions, P. L., Touzi, N. (1999) Applications of Malliavin calculus to Monte Carlo methods in finance, Finance and Stochastics, 3(4) 391-412.

[4] Glasserman, P. (2003) Monte Carlo Methods in Financial Engineering, Springer.

[5] Glasserman, P., Liu, Z. (2007) Sensitivity estimates from characteristic functions, Proceedings of the 39th conference on Winter simulation.

[6] Glasserman, P., Liu, Z. (2010) Estimating Greeks in simulating Lévy-driven models, Journal of Computational Finance, 14(2) Winter.

[7] Grigelionis, B. (1999) Processes of Meixner type, Lithuanian Mathematics Journal, 39(1) 33-41.

[8] Grigoletto, M., Provasi, C. (2009) Simulation and estimation of the Meixner distribution, Communications in Statistics - Simulation and Computation, 38(1) 58-77.

[9] Houdré, C., Kawai, R. (2007) On layered stable processes, Bernoulli, 13(1) 252-278.

[10] Imai, J., Tan, K.S. (2009) Dimension reduction approach to simulating exotic options in a Meixner Lévy market, IAENG International Journal of Applied Mathematics, 39(4) 265-275.

[11] Kawai, R., Kohatsu-Higa, A. (2010) Computation of Greeks and multidimensional density estimation for asset price models with time-changed Brownian motion, Applied Mathematical Finance, 17(2) 301-321.

[12] Kawai, R., Takeuchi, A. (2011) Greeks formulas for an asset price model with gamma processes, Mathematical Finance, 21(4) 723-742.

[13] Kawai, R., Takeuchi, A. (2010) Sensitivity analysis for averaged asset price dynamics with gamma processes, Statistics \& Probability Letters, 80(1) $42-49$.

[14] Kawai, R., Takeuchi, A. (2012?) Computation of Greeks for asset price dynamics driven by stable and tempered stable processes, Quantitative Finance, doi:10.1080/14697688.2011.589403.

[15] Kawai, R., Masuda, H. (2011) Local asymptotic normality property for Meixner Lévy processes with discrete observations, Statistics \& Probability Letters, 81(4) 460-469.

[16] Kawai, R., Masuda, H. (2011) On simulation of tempered stable random variates, Journal of Computational and Applied Mathematics, 235(8) 2873-2887.

[17] Madan, D.B., Yor, M. (2008) Representing the CGMY and Meixner Lévy processes as time changed Brownian motions, Journal of Computational Finance, 12(1)

[18] Rosiński, J. (2007) Tempering stable processes, Stochastic Processes and their Applications, 117(6) 677-707.

[19] Schoutens, W., Teugels, J.L. (1998) Lévy processes, polynomials and martingales, Communications in Statistics: Stochastic Models, $14,335-349$.

[20] Yano, K., Yano, Y., Yor, M. (2009) On the laws of first hitting times of points for one-dimensional symmetric stable Lévy processes, Séminaire de Probabilités XLII, 187-227, Lecture Notes in Math., 1979, Springer, Berlin. 